\title{
Atresia of left atrioventricular orifice
}

Sir,

In their paper (Br Heart $\mathcal{f}$ 1981; 45: 393-401) Thiene et al. state that in order to avoid confusion and discrepancies in nomenclature with regard to the terms "mitral" and "tricuspid" atresia they prefer to distinguish between atresia of left and right atrioventricular orifices. This implies that cardiac malformations of different pathogenesis are lumped together. If this is overlooked one might erroneously assume that natural entities are classified. Morphological characteristics and spatial relations inherent in the asymmetrical development of the morphological right and left side of the heart are neglected. The left to right asymmetry of the ventricular part of the heart, to which valve morphology is always secondary, is concealed by describing structures as left and right hand instead of as morphologically left and right.

It is not always easy to distinguish between the morphological left and right side of the heart, but quite a number of features can be used as guidance, for example the ventricular morphology, the position of the conducting system, the relation of the septum to the crux, and the position of a rudimentary chamber. The atretic left atrioventricular orifice may be either the mitral or the tricuspid orifice, dependent on the bulboventricular loop during cardiac development.

In our opinion the nomenclature now proposed by Thiene et al. unnecessarily simplifies terminology to such an extent that the material becomes less accessible to those who try to understand the developmental background of cardiac malformations. They simplify morphology itself, which cannot be done without causing confusion.

\section{Adriana C Gittenberger-de Groot and}

Arnold C G Wenink, Anatomisch-Embryologisch Laboratorium der Rijksaniversiteit, Leiden, Netherlands.
This letter was shown to Dr Thiene and his coauthors who reply as follows:

Sir,

We must admit to being confused as to the precise problem encountered by Drs Gittenberger-de Groot and Wenink. We were at pains to point out in our introduction the problems inherent in the use of "mitral" and "tricuspid" atresia. We then emphasised that the hearts studied were unified by the fact that they had no direct communication between the morphologically left atrium and the ventricular mass. In terms of left atrial morphology the hearts then are very much a "natural entity", accepting that there is a difference between an imperforate valve and an absent connection (see below). Drs Gittenberger-de Groot and Wenink suggest that we describe structures as left and right hand rather than as morphologically left and right. Fig. 1 of our paper gave a clear account of all the hearts studied, describing how the morphologically right atrium was connected in some hearts to morphologically right ventricular chambers, and in other hearts to morphologically left ventricular chambers. This Figure also illustrated the important variation in relation of a rudimentary right ventricular chamber when the right atrium connected to a morphologically left ventricular chamber. We described the relation of a septum to the crux of the heart in all the hearts categorised as "univentricular". In other words, we described all the features they discuss in their second paragraph apart from the position of the conduction system. We also pointed out how in some hearts the atresia was the result of an imperforate valve membrane and in others the result of absence of the left atrioventricular connection.

We submit, therefore, that we described precisely the anatomy of the hearts studied. Drs Gittenbergerde Groot and Wenink apparently criticise us for not describing the presumed anatomy of a structure that is not there; that is the absent left atrioventricular connection. Our introduction and discussion were designed to show how the problems in nomenclature devolve from overemphasis upon embryology, this itself derived from presumptions concerning non107 
existent structures. Apparently the Leiden workers wish to perpetuate this confusion. If so, then all the information they require to make their presumptions is clearly stated in our paper. We wished to remove such confusion and believe we have done so. We strongly contest their claim that we have simplified morphology. All we have done is to describe morphology as it is observed.
G Thiene,

$\mathrm{R} H$ Anderson, and

F J Macartney,

The University of Padova,

Italy; Institute of

Child Health, London;

and the Cardiothoracic

Institute, Brompton Hospital, London. 